\title{
Atypical, ventro-ventral copulation position in aphids of the genus Stomaphis Walker, 1870 (Insecta, Hemiptera)
}

\author{
Łukasz Depa ${ }^{1,2}$, Mariusz Kanturski ${ }^{1}$, Artur Taszakowski ${ }^{1}$, Karina Wieczorek ${ }^{1}$ \\ ${ }^{1}$ Zoology Department, Faculty for Biology and Environmental Protection, University of Silesia Bankowa 9, 40-007 \\ Katowice, Poland \\ ${ }^{2}$ E-mail: lukasz.depa@us.edu.pl
}

Key words: Aphididae, dwarfism, mate guarding, sexual competition, speciation

\begin{abstract}
Firm matching of genitals during copulation is of critical importance to effective insemination and thus, gene flow. During the evolution of insects, an effective position during copulation promoted higher fecundity through control over the act of mating or elimination of competitors. Usually during insect copulation, either twisting or flexing of the male abdomen occurs, and genitals remain symmetrically or asymmetrically disposed following changes in the mating position. However, it is always the dorsal side of the male genitalia that makes contact with the ventral side of female abdomen. Here we present the unusual case of a 'belly-to-belly' copulation, with symmetrically positioned male genitals and no twisting of the abdomen. During the mating of two species in the Stomaphis genus of large, tree dwelling aphids, the dwarfish male is attached to the underside of the female, with the ventral part of its genitals contacting the ventral part of female abdomen, and the aedeagus effectively inserted into the female genital organs. Interestingly, congeneric species do not exhibit this sort of mating, but differences in the genital plates of females, between species, may play an important role. These observations raise many questions concerning the possible dominant role of the female during mating and later, during mate guarding by male, which can lead to monandry in this generally polyandrous group of insects. It is possible that this sort of mating is either an adaptation to the competitive behaviour of other males or a consequence of the obligatory mutualistic relationship with ants, and the adaptation to specialised ecological niches enforced by this relationship. If ants do influence the mating habits of Stomaphis then it is possible that speciation in this group of insects, and phytophagous insects generally, is partially driven by their relationship with ants.
\end{abstract}

\section{Content}

Introduction

Material and methods

Results

Discussion

Acknowledgements

References 182

\section{Introduction}

A dorso-ventral copulation position is the norm in insects, with the male positioned above the female and the distal part of its abdomen bent towards the genital pore of the female sexual organs, which feature various adaptive modifications (Huber, 2010). Very rarely a ventro-ventral mating position has been observed, involving twisting of the male abdomen or asymmetry of the male external genitalia (Huber et al., 2007). Special appendages of the male external genitalia help to direct the aedeagus (the membranous part of the phallus) into the genital pore of the female.

The conjunction between the genital structures of both partners should be very precise, preventing unexpected separation, e.g. when the mating takes place during flight. Thus the correct copulatory position is directly related to the structure of genitalia, and any incorrect positioning of the phallus may result in disrupted insemination of the female, although asymmetrically positioned genitalia may themselves be an adaptation for effective copulation and are a recurrent phenomenon in many insect taxa (Huber et al., 2007; Schilthuizen, 2007; Lang and Orgogozo, 2012; Breeschoten et al., 2013).

The external genitalia of male aphids have been extensively studied by Wieczorek et al. $(2011,2012)$ and serve as a good indicator of species identity among these insects. Aphids breed sexually in the autumn, with males occurring only briefly at the end of the season. As a consequence there are few studies of mating in this group of insects (Dixon, 1998).

Here we present the unusual case of an 'under-female' (ventro-ventral) or 'belly-to-belly' (Huber et al., 2007) mating position for two species of male Stomaphis Walker, 1870 (Hemiptera, Aphididae, Lachninae). These observations showed no evidence of twisting of 


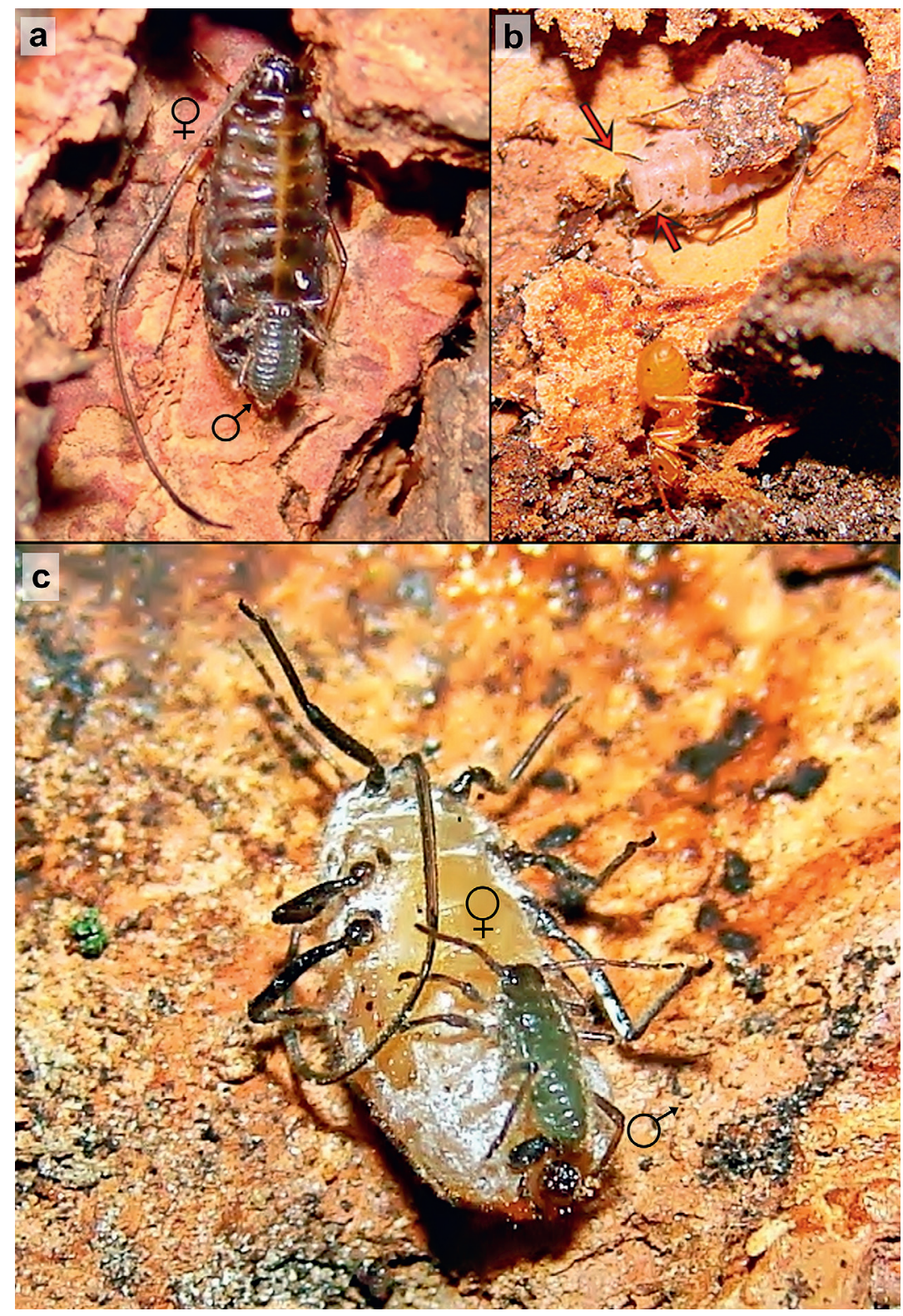

Fig. 1. Dorso-ventral vs ventro-ventral copulatory position in Stomaphis species. a, typical for insects copulatory position in $S$. quercus; b, oviparous female of $S$. graffii with only legs visible (arrows) of copulating male; c, atypical copulatory position in S. longirostris. the male abdomen or asymmetrically disposed genitalia, and are unique among insects.

Female Stomaphis are among the largest aphids, with body lengths reaching ca. $8 \mathrm{~mm}$. In contrast, Stomaphis males are very small (dwarfish), with a body length of ca. $2.1-3.3 \mathrm{~mm}$. Despite the huge size of females, they tend to feed in discreet positions, hidden in bark crevices and covered with soil by ants. Consequently many Stomaphis species are rarely found and research concerning their biology and ecology is difficult to undertake. All the known Stomaphis species are strongly dependent on their mutualistic relationship with ants (Depa, 2012, 2013; Depa et al.,2012) and the life mode of the aphids closely follows the life mode of their ant partner. Thus the species showing atypical copulation and a cryptic life mode subject of this study are associated with the ant Lasius brunneus which typically nests in old tree trunks

The aim of this study was to describe an atypical mode of copulation in some species of Stomaphis and to consider how genital morphology and a mutualistic relationship with ants may have influenced the development of this phenomenon. 


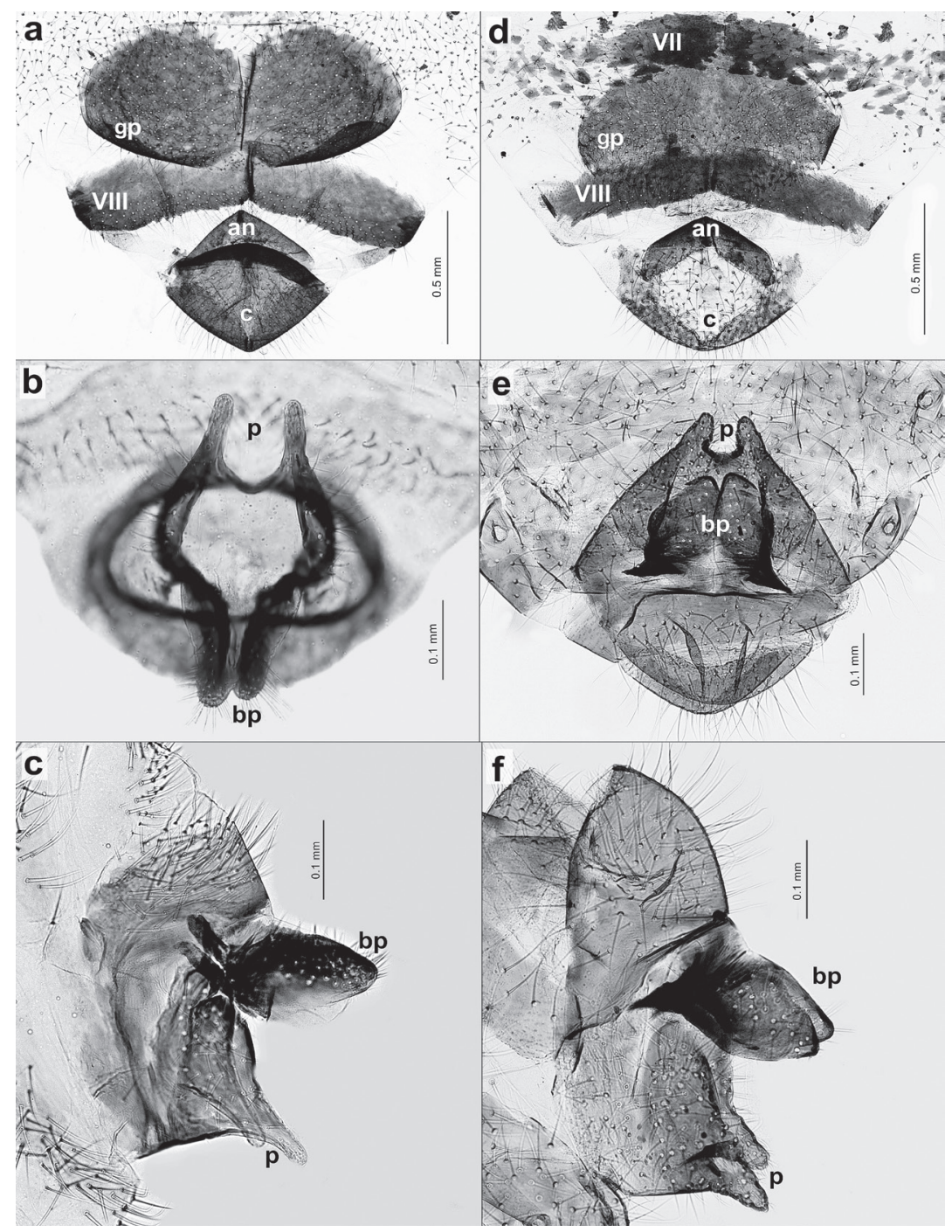

Fig. 2. Comparison of the oviparous female's genital plate and the external genitalia of male of S. longirostris-group (a-c) and S. quercus-group (d-f). a, divided genital plate (gp) positioned above anal plate (an) and cauda (c) in S. graffii (ventral view); $\mathrm{b}$, c, elongated, finger-like projections of parameres (p), positioned above smooth basal part of the phallus (bp) in $S$. graffii (b, ventral view, c, lateral view); d, not divided genital plate (gp) positioned above anal plate (an) and cauda (c) in S. quercus (ventral view); e, f, very short projections of parameres (p), positioned above clubshaped basal part of the phallus (bp) in $S$. quercus (e - ventral view, f - lateral view).

\section{Material and methods}

Regular field studies of the sexual generations of Stomaphis were carried out in the south of Poland between 2010 and 2013, and between mid-August and mid-November. Copulating adult males and oviparous females of 4 species were observed: $S$. graffii Cholodkovsky, 1894 (N: 5057'13.45”, E: 18²6’9.69”, 4.11.2011, N: 4937'39", E: $\left.21^{\circ} 19^{\prime} 4622.10 .2013\right) ;$ S. longirostris (Fabricius, 1787) (N: 50²3'45”, E: 1857'10”, 28.09.2011); $S$. quercus (Linnaeus, 1758) (N: 506'7.48”' E: 2149'31.13", 09.10.2010); S. wojciechowskii Depa, 2012 (N: 506'15.82" E: 2149'41.73", 22.10.2013).

The specimens were preserved in $70 \%$ ethyl alcohol, examined and photographed using a stereoscopic mi- croscope Olympus SZH10. Field photographs were taken with a Sony SLT a37 digital camera and Sigma $50 \mathrm{~mm}$ macro lens. Microscope slides were prepared for correct identification of species and subsequently deposited in the entomology collection of the Department of Zoology, University of Silesia, Katowice, Poland (UŚ), slides no: UŚ S42.2/2011, UŚ S22.10.A/2013, UŚ S28.09/2011, UŚ S30.1/2010, UŚ S22.10B/2013.

\section{Results}

In September 2011 field observations were made of atypical copulation in $S$. longirostris. The oviparous female was observed to roll onto its dorsum, revealing 


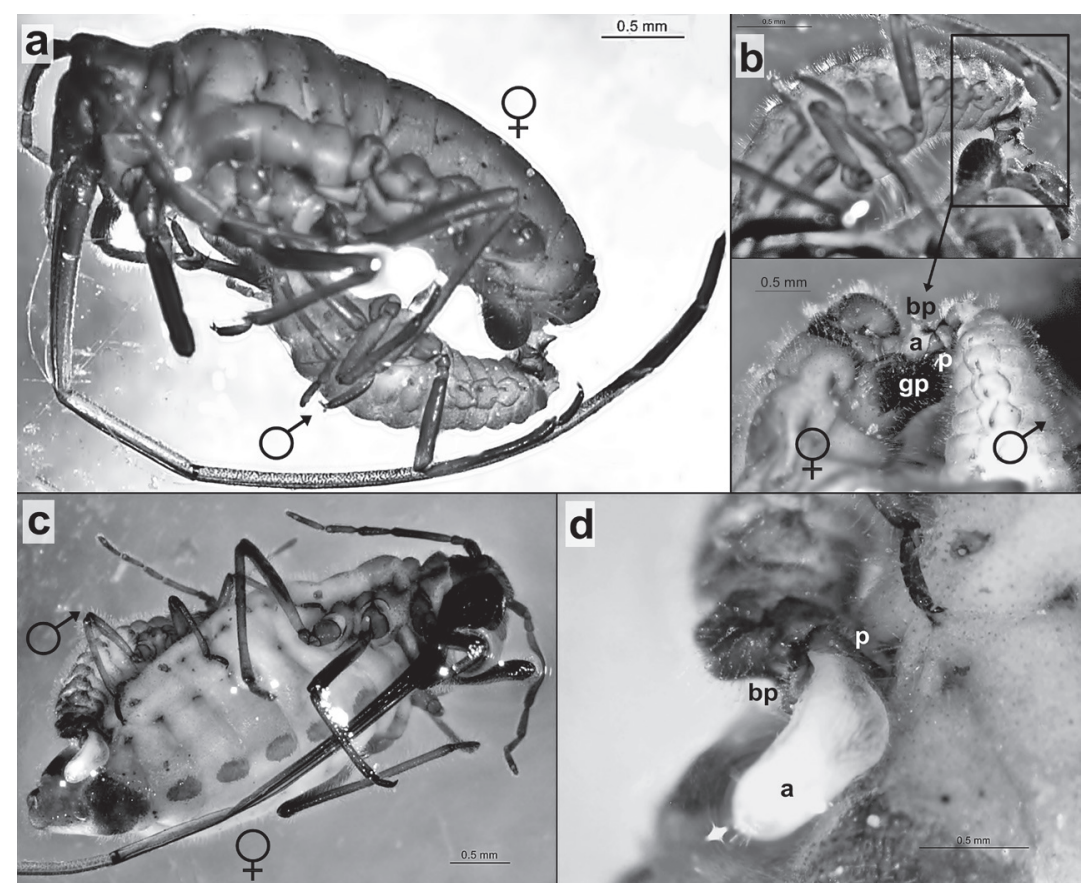

Fig. 3. Atypical and typical position of copulating pairs of Stomaphis. a, individuals of $S$. graffii in atypical ventro-ventral copulatory position (preserved in $70 \%$ ethanol); b, close up of the ventro-ventral copulatory position $S$. graffii: elongated projections of parameres (p) penetrate the divided genital plate ( $\mathrm{gp}$ ) of oviparous female while basal part of the phallus (bp) serves to keep aedeagus (a) inserted inside female body; c, typical copulatory position/ mate guarding in S. wojciechowskii (preserved in $70 \%$ ethanol); d, sclerotised basal part of the phallus (bp) and parameres (p) creating an obtuse angle, sustaining proximal part of the enormous aedeagus (a). the copulating male beneath (Fig. 1c).

A second observation in November, 2011 concerned aphids of the closely related species $S$. graffii. Detailed study of field photographs of oviparous females of $S$. graffii revealed the presence of males underneath the oviparous female, with only their middle and hind legs visible (Fig. 1b). Most copulating pairs of S. longirostris and $S$. graffii separated after collection, probably due to shrinkage by dehydration in the alcohol. However, in October 2013 a single pair of copulating S. graffii did not separate during transfer to the laboratory. Detailed analysis of the external genitalia led to the following, tentative conclusions about the ventro-ventral copulation positions in the species studied (S. longirostris-group), and the role of the genital structures:

1. The posterior part of the male abdomen was only slightly bent towards the genital pore of the female (Fig. 3a).

2. The sclerotized, basal part of the phallus and parameres were clearly visible, not inserted inside the genital pore of the female, but projecting outwards creating an obtuse angle, sustaining the position of the aedeagus (Fig. 3b).

3. The genital plate is divided into two separate sclerotized subplates which were bent downwards and slightly sideways (Fig. 2a).

4. Elongated, finger-like projections of the parameres, positioned above the basal part of the phallus (Fig. $2 \mathrm{~b}, \mathrm{c}$ ), penetrated the divided genital plate of the oviparous female, while the sclerotised, basal part of the phallus served to keep the aedeagus inserted inside the female (Fig. 3b).

5. There was no twisting of male external genitalia to match those of the female. The orientation of male genitalia during copulation was exactly the same as when at rest.

Additional observations of the more typical, dorsoventral copulation position concerned the closely related species $S$. quercus and $S$. wojciechowskii ( $S$. quercus-group), and enabled the following conclusions to be drawn:

1. The male, or sometimes males, approached and climbed onto the giant female body from behind (Fig. 1a).

2. The posterior part of the male abdomen was bent towards the genital pore of the female (Fig. 3c).

3. The genital plate of oviparous females constituted a single sclerotic plate (Fig. 2d).

4. The projections of parameres, positioned above the basal part of the phallus, were very short (Fig. 2e, f).

5. The sclerotised, basal part of the phallus and parameres were clearly visible, projecting outwards and creating an obtuse angle, sustaining the proximal part of the large (ca. $1.10-1.32 \mathrm{~mm}$ ) aedeagus (Fig. 3d). 


\section{Discussion}

Male dwarfism and a cryptic life mode may play a role in determining the nature of the copulation behaviour observed in the two groups of Stomaphis. In general terms little is known about the mating behaviour of aphids, but Stomaphis can be distinguished from other aphid groups by many differences in their life cycle and morphology. Feeding on tree trunks results in modified mouthparts, with lengthened stylets and rostrum - a phenomenon known to have occurred in tree feeding aphids since at least the Lower Cretaceous (Heie and Azar, 2000; Wegierek and Grimaldi, 2010; Homan and Wegierek, 2011). In the case of Stomaphis this feeding habit has also resulted in a large body size (Dixon, 1998). A similar morphological adaptation comprising a rostrum significantly longer than body is observed in larvae of the unrelated genus Prociphilus (Eriosomatinae), which feed on the bark crevices of Abies roots. However, Prociphilus aphids have not developed an especially large body size and do not depend on mutualistic relationship with ants (Heie, 1980), possibly because they are covered with a protective layer of wax.

With their stylets deeply embedded in host tissues during feeding, Stomaphis are unable to escape rapidly from predators or parasitoids. Extruding stylets from a tree takes at least a few minutes, because the rostrum which is invaginated into the abdominal cavity, also needs to be extruded. By forming close associations with mutualistic ants, Stomaphis may secure a degree of protection from natural enemies. Survival without mutualistic ants may be no more than a few days (Lorenz and Scheurer, 1998).

There are two dominant species of Lasius ants in Europe associated with Stomaphis: L. fuliginosus and L. brunneus. Both of these species have arboreal but quite distinctive life modes. L. fuliginosus is an aggressive, above surface forager, whilst L. brunneus is a timid and cryptic ant, foraging mainly on trees, very often in bark crevices covered by soil, and is rather submissive to other ant species (Czechowski et al., 2012). However, the latter is much more common in parts of Europe and is the dominant ant associated with S. longirostris and S. graffii, with L. fuliginosus most often associated with S. quercus (Depa et al., 2012).

The habitat of $S$. longirostris and $S$. graffii associated with L. brunneus is unusual, and may be compared to living underground or being enclosed in a gall. There is very little space in ant chambers under bark and there is no light. Males have a much reduced morphology with no mouthparts, greatly reduced eyes and only basic olfactory organs on their antennae. They do not need to look long and hard for a mate, given the small, enclosed environment of the ant chamber - a small area under the bark, connected by tunnels with the main nest, where the ant brood is kept.

It is possible that the mating behaviour of the $S$. longirostris-group is an adaptation to avoiding interference with or becoming stuck to the droplets of honeydew, excreted by ant attended females, or to avoid being crushed by the chamber vault, though there remains a risk of being crushed by the female against the 'chamber bed'. The extent to which the female influences the mating behaviour or cooperates with the male is not known, but may play a role in this behaviour (Huang and Caillaud, 2012; Kvarnemo and Simmons, 2013).

A further possibility is that the behaviour observed is some sort of mate guarding or marking. In aphids this is a rarely observed and poorly studied phenomenon, which occurs in the unrelated species Pemphigus spyrothecae (Eriosomatinae) (Dixon, 1998) and in the closely related aphid genera Cinara and Lachnus (Dagg and Scheurer, 1998). Being well hidden under the female during copulation prevents aggression by competing males, and competitive copulation by other males.

Being enclosed in a small ant chamber, males may encounter very high levels of intraspecific competition from other males, which may not necessarily be of the same genetic lineage, since limited dispersal by alate females occurs in these aphids (Depa, 2012, 2013; Takada, 2008). As a single aphid colony may last at least as long as its protective ant colony (e.g. 20 years or so) there is a good chance of that colony incorporating a few migrant, alate females during that time. Equally it is possible that a few unrelated males will compete for access to oviparous females. However, due to their cryptic life mode, very little is known about the male/ female ratio in the colony (Hales et al., 2007), which may further affect the competitive behaviour of males and mate guarding. For males in the S. longirostris group, mate searching, with its associated energy expenditure and predation risk, is probably much less important than it is in the more mobile $S$. quercus. The ventro-ventral position during copulation with the male hidden under the oviparous female, might be a specific form of mate guarding, which probably evolved in response to the specialised ecological niche occupied by these aphids, and can lead to monandry (Kvarnemo and Simmons, 2013).

The proposed course of development for this behaviour is as follows. In the small space of the ant chamber males, who have never been observed to leave the nest 
of their attendant ant, are forced to compete for access to oviparous females. If a few males try to mate with a single female, the one that manages to hide under it may be able to guard its mate more effectively, being closer to the genital pore. Simultaneously, males trying to copulate in a dorso-ventral position may have lower fitness, due to increased mortality through crushing on the chamber vault or by being stuck on excreted honeydew.

In a longer evolutionary perspective, this behaviour may be considered partly as an adaptation to feeding on tree trunks, and partly as a result of occupying a specific ecological niche created by the mutualistic partner - ants. On this basis it is possible to speculate that speciation in phytophagous insects may in some circumstances be driven by factors other than host plant selection (Berlocher and Feder, 2002; Bolnick and Fitzpatrick, 2007). In the case of aphids that are obligate myrmecophiles, their life mode must closely follow that of their ant host. However, within the range of single aphid population, there may occur sub-populations living with ants of a different biology and that therefore require different life modes for aphids such as living on the tree surface (open life mode) or under the bark (cryptic life mode). Adaptation to living in the closed chambers of ant nests helped to develop the particular mode of copulation in the group of Stomaphis described in this paper, as opposed to Stomaphis living with ants having an open life mode, when males may copulate while being over the female, without significant risk of being injured. Different modes of living promote different sexual behaviour and may also disturbed gene flow if the competitive behaviour of ants disables the mixing of aphids attended by different ant species. The occupation of a very specialised ecological niche (in this case literally the place of living - a spatially limited ant chamber) may serve as a selective factor of speciation, where a single population diverges into two populations, both adapted to a mutualistic association with two different ant species. This process may also have affected other honeydew producing hemipterans such as coccids (Coccoidea), some Tettigometridae (Fulgoromorpha) and Membracidae (Cicadomorpha), all of which are phytophagous and may have mutualistic relationships with ants.

A question which needs further investigation is what the selective mechanism is promoting male dwarfism in Stomaphis. Male Stomaphis do not feed or produce honeydew so dwarfism may be a collateral effect of not being a trunk-feeder or a partner in the mutualism with ants. This may have resulted in a scaling down of male morphology but further work is necessary to determine the factors involved.

\section{Acknowledgements}

Authors would like to thank Dr. Roger L. Blackman for his helpful comments on the first version of the manuscript. We would also like to express our gratitude to Prof. Dr. Michael Schmitt, and an anonymous referee for providing valuable comments and suggestions which helped to improve the manuscript. Finally, we thank Ed Baker for linguistic assistance with the manuscript.

\section{References}

Berlocher SH, Feder JL. 2002. Sympatric speciation in phytophagous insects: moving beyond controversy? Annual Review of Entomology 47: 773-815.

Bolnick DI, Fitzpatrick BM. 2007. Sympatric speciation: models and empirical evidence. Annual review of Ecology, Evolution and Systematics 38: 459-487.

Breeschoten T, Clark DR, Schilthuizen M. 2013. Evolutionary patterns of asymmetric genitalia in the beetle tribe Cyclocephalini (Coleoptera: Scarabaeidae: Dynastinae). Contributions to Zoology 82: 95-106.

Czechowski W, Radchenko A, Czechowska W, Vepsäläinen K. 2012. The ants of Poland with reference to the myrmecofauna of Europe. Fauna Poloniae, Museum and Institute of Zoology, Polish Academy of Science, Warsaw.

Dagg JL, Scheurer S. 1998. Observations on some patterns of the males' sexual behaviour of certain aphid species. In: JM Nieto Nafria \& AFG. Dixon (Eds.) Aphids in natural and managed ecosystems: 167-171. Universidad de León: Secretariado de publicaciones.

Depa Ł. 2012. Abundance of Stomaphis graffii Cholod. (Hemiptera) on maple trees in Poland. Central European Journal of Biology 7: 284-287.

Depa Ł. 2013. Life cycle of maple-tree aphid Stomaphis graffii Cholodkovsky, 1894 (Hemiptera, Aphididae). Animal Biology 63: 313-320.

Depa Ł, Mróz E, Szawaryn K. 2012. Molecular identity of $S$. quercus and description of a new species. European Journal of Entomology 109: 435-444.

Dixon AFG. 1998. Aphid ecology. An optimization approach. London: Chapman \& Hall.

Hales DF, Tomiuk J, Wöhrmann K, Sunnucks P. 1997. Evolutionary and genetic aspects of aphid biology: a review. European Journal of Entomology 94: 1-55.

Heie OE. 1980. The Aphidoidea (Hemiptera) of Fennoscandia and Danmark. I General Part. The Families Mindaridae, Hormaphididae, Thelaxidae, Anoecidae and Pemphigidae. Fauna Entomologica Scandinavica vol. 9: 1-236.

Heie OE, Azar D. 2000. Two new species of aphids found in Lebanese amber and a revision of the family Tajmyraphididae Kononova, 1975 (Hemiptera, Sternorrhyncha). Annals of the Entomological Society of America 93: 1222-1225.

Homan A, Wegierek P. 2011. A new family of aphids (Hemiptera, Sternorrhyncha) from the Lower Cretaceous of Baissa, Transbaikalia. Zookeys 130: 167-174.

Huang MH, Caillaud MC. 2012. Inbreeding avoidance by recognition of close kin in the pea aphid, Acyrthosiphon pisum. Insect Science 12: 39. [available on: insectscience.org/12.39] 
Huber BA, Sinclair BJ, Schmitt M. 2007. The evolution of asymmetric genitalia in spiders and insects. Biological Reviews 82: 647-698.

Huber BA. 2010. Mating positions and the evolution of asymmetric insect genitalia. Genetica 138: 19-25.

Kvarnemo C, Simmons LW. 2013. Polyandry as a mediator of sexual selection before and after mating. Philosophical Transaction of the Royal Society B 368: 20120042. doi: 10.1098/rstb.2012.0042

Lang M, Orgogozo V. 2012. Distinct copulation positions in Drosophila pachea males with symmetric or asymmetric external genitalia. Contributions to Zoology 81: 87-94.

Lorenz H, Scheurer S. 1998. Biology and generation-order of Stomaphis quercus (Lachnidae) living on Betula pendula near Berlin, Germany. Pp. 243-250 in: JM Nieto Nafria, AFG. Dixon, eds, Aphids in natural and managed ecosystems. Universidad de León: Secretariado de publicaciones.

Schilthuizen M.2007. The evolution of chirally dimorphic insect genitalia. Tijdschrift voor Entomologie 150: 347-354.
Takada H. 2008. Life cycles of three Stomaphis species (Homoptera: Aphididae) observed in Kyoto, Japan: possible host alternation of S.japonica. Entomological Science 11: 341-348.

Wegierek P, Grimaldi DA. 2010. A new subfamily of aphids (Hemiptera, Aphidomorpha) from the early Cretaceous Lebanese amber with a description of the oldest apterous morphs. Acta Geologica Sinica 84: 665-672.

Wieczorek K, Płachno BJ, Świątek, P. 2011. A comparative morphology of male genitalia of Aphididae (Insecta, Hemiptera): part 1. Zoomorphology 130: 289-303.

Wieczorek K, Płachno BJ, Świątek P. 2012. A comparative morphology of male genitalia of Aphididae (Insecta, Hemiptera): part 2. Zoomorphology 131: 303-324.

Received: 15 January 2014

Revised and accepted: 30 April 2014

Published online: 6 June 2014

Editor: M. Schilthuizen 